\title{
Amlodipine-Induced Gingival Overgrowth: A Case Report
}

\author{
Yogesh Upadhyay \\ Chandra Dental College And Hospital., Safedabad,Barabanki. U.P, India
}

\begin{abstract}
Gingival overgrowth is frequently observed in patients taking certain drugs such as calcium channel blockers, anticonvulsants and immunosuppressant. This can have a significant effect on the quality of life as well as increasing the oral bacterial load by generating plaque retention sites. Amlodipine, a third generation calcium channel blockers has been shown to promote gingival overgrowth although in very limited cases reported. The management of gingival overgrowth seems to be directed at controlling gingival inflammation through a good oral hygiene regimen. However in severe cases, surgical excision is the most preferred method of treatment, followed by rigorous oral hygiene procedures. This case report describes the management of gingival overgrowth in a hypertensive patient taking amlodipine. Combination of surgical gingivectomy and $\mathrm{CO} 2$ laser treatment was used to remove the gingival overgrowth. CO2 laser surgery produced good haemostasis and less pain during the procedure and post operatively. This case report has also shown that endodontic treatment alone without a change in associated drug can yield satisfactory clinical response.
\end{abstract}

Keywords: Calcium channel blocker, CO2 laser, drug-induced, gingival overgrowth, gingivectomy

\section{Introduction}

Drug induced gingival overgrowth (GO) is frequently observed as a side effect with the use of several medications in the susceptible patients. Medication mainly implicated are the anticonvulsant such as phenytoin for treatment to control seizure disorders in epileptic patient, calcium channel blockers (CCB) such as nifedipine for treatment of hypertension or angina pectoris immunosuppressant such as cyclosporine A for treatment to prevent rejection in patient received organ transplant (Seymour et al., 1996).

Many reports had discussed patients taking nifedipine induced GO. During the past few years amlodipine has been used with increasing frequency and also has been reported to promote GO (Seymour et al., 1994). Recently, Lafzi et al. (2006) had reported rapidly developed gingival hyperplasia in patient received 10 mg per day of amlodipine within two months of onset. Amlodipine, a dihydropyridine derivative is a third generation of calcium channel blockers which shown to have longer action and weaker side effect compared to the first generation such as nifedipine (Ellis et al., 1993). The prevalence of GO in patients taking amlodipine was reported to be 3.3\% (Jorgensen, 1997) which is lower than the rate in patients taking nifedipine, $47.8 \%$ (Nery et al., 1995).

The clinical features of GO usually presented as enlarged interdental papillae and resulting in a lobulated or nodular morphology (Hallmon and Rossmann, 1999). The effects normally limited to the attached and marginal gingivae and more frequently observed anteriorly. Histologically, in nifedipine-induced gingival overgrowth it was described as thickening of the spinous cell layer, slight to moderate hyperkeratosis, fibroblastic proliferation and fibrosis of lamina propria (Hallmon and Rossmann, 1999). In this case report, we treated severe GO in patient taking amlodipine for treatment of hypertension. The management consists of oral hygiene procedures and combination of surgical gingivectomy and $\mathrm{CO} 2$ laser treatment.

\section{Case Report}

A 55-year old woman was referred to the Department of Conservative \& Endodontics, Faculty of Dentistry, Chandra Dental College \& Hospital, Barabanki, complaining of swellings on her gingiva for several months induration. She felt very uncomfortable as the swelling interfered while chewing and sometimes there was bleeding spontaneously. She had hypertension since few years and was on medications i.e. Amlodipine $5 \mathrm{mg}$ daily, Metoprolol 100mg daily, Lovastatin as an adjunct to cholesterol control and Aspirin $75 \mathrm{mg}$ daily.

Generally she looked well and alert. Intra-orally, there was massive GO on the labial/ palatal of the upper and lower teeth with less pronounced at the lower right quadrant. The interdental papillae were inflamed and lobulated in appearance mainly at the lower anterior teeth (Figure 1). Her oral hygiene was very poor with abundant plaque and calculus. Bleeding on probing was detected on all affected areas. There were multiple retained roots embedded in the overgrown tissues of the upper arch (Figure 2). Periodontal pockets were 3 to 9 $\mathrm{mm}$ characterized more of pseudo pockets. Her upper left central incisors and canine were deeply carious. Tooth 38 was mobile grade I with Class III furcation involvement and a few teeth were missing. The clinical diagnosis was drug-induced gingival overgrowth. 


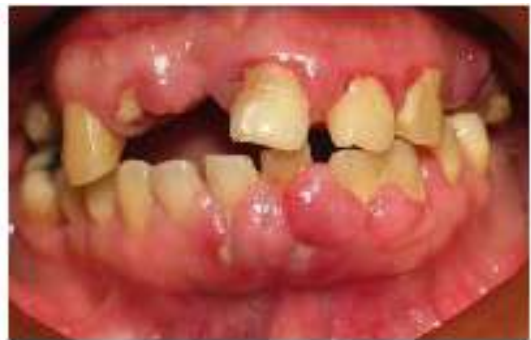

Figure 1 Intraoral picture showing the gingival overgrowth at the initial appearance

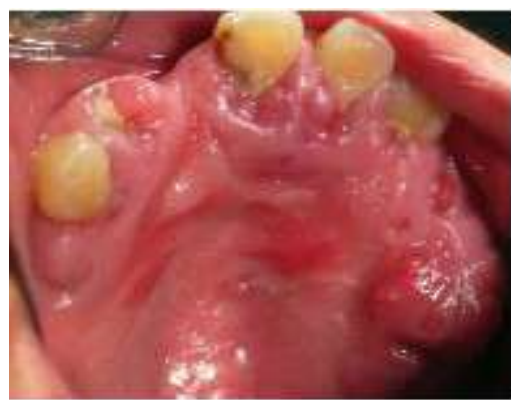

Figure 2 Gingival appearance at the palatal region

Full mouth scaling and polishing of all teeth was done and patient was given oral hygiene instruction and motivation at the first visit.

Review after one week revealed some reduction of the GO particularly at the upper arch. All multiple roots were then extracted under local anaesthesia. At the following visit, surgical and laser gingivectomy was performed for the lower unwanted gingiva. The overgrown tissue was resected by using scalpel blade size 15 . Surgical site was then treated with a super pulsed wave mode CO2 laser (Luxar Navopulse, Boston, USA) set at 5 watts (Figure 3). The charred layer produced by lasering acts as protective barrier and was not removed after this procedure. Four weeks later the same procedure was done for the upper GO. All procedures were carried out under local anaesthesia.

Few pieces of enlarged tissue from the labial part of the teeth 31, 32 and the palatal part of tooth 21 were sent for histopathological examination (HPE). Patient was prescribed tablet paracetamol $1 \mathrm{~g}$ for three days and mouthwash Chlorhexidine Gluconate $0.12 \%$ for two weeks after each surgical procedure. The HPE demonstrated an irregular fibrous overgrowth composed of collagenous connective tissues with a diffuse chronic inflammatory cell infiltrate and covered by an intact hyperparakeratotic and acanthotic stratified squamous epithelium.

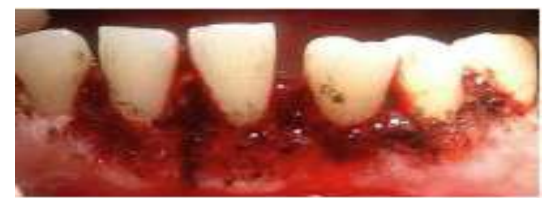

Figure 3 Surgical gingivectomy and $\mathrm{CO} 2$ laser treatment on the lower buccal gingiva. Note that charred layer and less bleeding after the procedure

Follow up was done one to three monthly. Upon examination at 3 month review, the periodontal pockets were generally reduced to $3 \mathrm{~mm}$. Very mild gingivitis was observed at the labial surface of lower incisors. Regular oral hygiene reinforcement and scaling was done for her. Two years after completion of the surgery, disappearance of GO and satisfactory periodontal condition were confirmed (Figure 4). Patient was then referred to Prosthodontist for the construction of prosthesis.

Removable partial over denture was planned with the teeth 13 and 22 served as the abutments. Teeth 21 and 38 were extracted due to poor prognosis. Elective endodontic was done for teeth 13 and 22. Both teeth were then decoronated at supra gingival level and the canal opening was sealed with amalgam filling. Over denture was then issued with some occlusal adjustment done. At 6 month follow up the patient was still on amlodipine however the periodontal conditions appeared satisfactory and pleasing (Figure 5). 


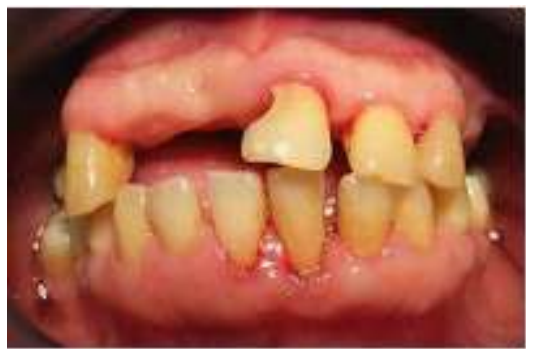

Figure 4 Gingival overgrowth had disappeared after two years of surgery

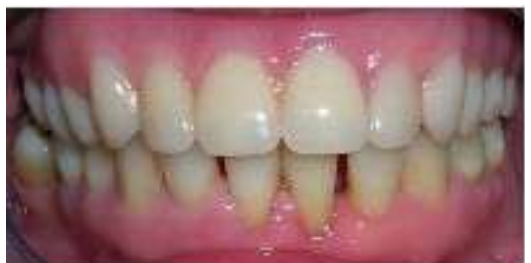

Figure 5 Intraoral view at sixth month review

\section{Discussion}

The pathogenesis of GO is uncertain and the treatment is still largely limited to the maintenance of an improved level of oral hygiene and surgical removal of the overgrown tissue. Several factors may influence the relationship between the drugs and gingival tissues as discussed by Seymour et al. (1996). Those factors were including age, genetic predisposition, pharmacokinetic variables, and alteration in gingival connective tissue homeostasis, histopathology, ultra-structural factors, inflammatory changes and drug action on growth factors. Most studies show an association between the oral hygiene status and the severity of drug- induced GO. This suggests that plaque-induced gingival inflammation may be important risk factor in the development and expression of the gingival changes (Barclay et al., 1992). In this present case the local environmental factors such as poor plaque control and multiple retained roots at the initial presentation may act as risk factors that had contributed to worsen the existing gingival enlargement and therefore complicate the oral hygiene procedures (Ikawa et al., 2002). There was some reduction of the overgrowth observed particularly at the upper arch after the initial therapy was advocated including extraction of the retained roots. Age is also an important risk factor for GO with particular reference to phenytoin and cyslosporin (Seymour, 2006) however is not applicable for CCB since the used of the drug is usually confined to the middle-aged and older adult (Seymour et al., 2000). The management of GO seems to be focusing at good oral hygiene regimen to control the gingival inflammation (Nery et al., 1995). The interaction between the drug and the gingival tissues could be enhanced by gingival inflammation caused by poor oral hygiene (Seymour, 1991). It has been shown that there was significant reduction of nifedipine-induced GO by thorough scaling and root planing and scrupulous plaque control (Hallmon and Rossmann, 1999). Surgical reduction of the overgrown tissues is frequently necessary to accomplish an aesthetic and functional outcome (Hallmon and Rossman, 1999). The treatment may consist of surgical gingivectomy and/ or laser gingivectomy. Laser is one of the most promising new technical modalities in endodontic treatment. The $\mathrm{CO} 2$ laser has a wavelength of $10,600 \mathrm{~nm}$, is readily absorbed by water and therefore very effective for the surgery of soft tissues, which have a high water content. Blood vessels in the surrounding tissues up to $0.5 \mathrm{~mm}$ are sealed (Aoki et al., 2004).

Thus the advantageous of laser over the scalpel are the strong haemostatic and bactericidal effect and provide a relatively dry field for improved visibility (AAP, 2002).

Discontinuation of the related drug has been shown to reduce the GO, however the growth will recurs when the drug was readministered (Lederman et al., 1984). In cases where alternate medication can be used, substitution in the related drug has been shown to result in regression of the overgrowth. Isradipine, a companion dihydropyridine calcium channel blocker has shown regression in about $60 \%$ of the GO previously induced by nifedipine (Hallmon and Rossman, 1999; Khera et al., 2005).

Another treatment modality that has been suggested was the use of topical application of folate solution on the GO. Drew et al. (1987) have demonstrated significant decreased of the GO when acid folic was topically applied on the phenytoin-induced gingival hyperplasia. Inoue and Harrison (1981) also found that folic acid supplementation decreases the severity of the GO. Phenytoin interferes with folic acid metabolism and lead to acid folic deficiency which is known to be associated with gingival inflammation. However there was no study reported the use of folic acid in the amlodipine- induced GO.

In this present case, gingival overgrowth was satisfactorily treated via initial periodontal therapy including oral hygiene instruction and motivation, followed with surgical gingivectomy and $\mathrm{CO} 2$ laser 
treatment. This case report also demonstrated that without a change in associated drug, endodontic treatment alone can yield satisfactory clinical response (Ikawa et al., 2002). As the periodontal condition was under control, prosthesis was constructed in order to fulfill the function and aesthetic of the patient. The prosthesis was designed to minimize the plaque retention sites.

However there is possibility for the GO to recur as long as the associated medication is continued and persistence with other risk factors (Mavrogiannis et al., 2006).

Therefore patient must be informed of this tendency and the importance of maintenance of the effective oral hygiene as key factors in preventing and managing gingival overgrowth associated with this drugs. Supportive follow up is necessary in an effort to monitor her gingival/ periodontal status, to assess and reinforce oral hygiene and to periodically provide professional care (Hallmon and Rossmann 1999) thus prevent the recurrence of GO.

\section{References}

[1] American Academy of Periodontology (AAP) (2002). Lasers in Periodontics. J Periodontol, 73: 1231-1239.

[2] Aoki A, Sasaki KM, Watanabe H and Ishikawa I (2004). Lasers in nonsurgical periodontal therapy. Perio $2000,36: 59-97$.

[3] Barclay S, Thomason JM, Idle JR and Seymour RA. (1992). The incidence and severity of nifedipine- induced gingival overgrowth. J Clin Periodontol, 19: 311-314.

[4] Drew HJ, Vogel RI, Molofsky W, Baker H and Frank O. (1987). Effect of folate on phenytoin hyperplasia. J Clin Periodontol, 14: 350-356.

[5] Ellis JS, Seymour RA, Thomason JM, Monkman SC and Idle JR (1993). Gingival sequestration of amlodipine and amlodipineinduced gingival overgrowth. Lancet, 341: 1102-1103.

[6] Hallmon WM and Rossmann JA (1999). The role of drugs in the pathogenesis of gingival

[7] overgrowth. A collective review of current concept. Perio 2000, 21: 176-196.

[8] Ikawa K, Ikawa M, Shimauchi H, Iwakura M and Sakamoto S (2002). Treatment of gingival overgrowth induced by manidipine administration: a case report. J Periodontol, 72: 115-122.

[9] Inoue F and Harrison J (1981). Folic acid and phenytoin hyperplasia. Lancet, 2: 86.

[10] Jorgensen MG (1997). Prevalence of Amlodipine- Related Gingival Hyperplasia. J Periodontol, 68: $676-678$.

[11] Khera P, Zirwas MJ and English JC (2005). Diffuse gingival enlargement. J Am Acad Dermatol, 52:491- 499.

[12] Lafzi A, Farahani RM and Shoja MA (2006). Amlodipine- induced gingival hyperplasia. Med Oral Patol Oral Cir Bucal, 11(6): E480-E482.

[13] Lederman D, Lumerman H, Reuben S and Freedman PD (1984). Gingival hyperplasia associated with nifedipine therapy. Report of a case. Oral Surg Oral Med Oral Pathol, 57: 620-622.

[14] Mavrogiannis M, Ellis JS, Thomason JM and Seymour RA (2006). The management of druginduced gingival overgrowth. J Clin Periodontol, 33: 434-439.

[15] Nery EB, Edson RG, Lee KK, Pruthi VK and Watson J (1995). Prevalence of nifedipine-induced gingival hyperplasia. $J$ Periodontol, 66: 572-578.

[16] Seymour RA (1991). Calcium channel blockers and gingival overgrowth. Br Dent J, 170: 376-379.

[17] Seymour RA, Ellis JS, Thomason JM, Monkman S, and Idle JR (1994).Amlodipine-induced gingival overgrowth. J Clin Periodontol, 21: 281-283.

[18] Seymour RA, Thomason JM and Ellis JS (1996). The pathogenesis of drug-induced gingival overgrowth. J Clin Periodontol, 23: $165-175$.

[19] Seymour RA, Ellis JS and Thomason JM (2000). Risk factors for drug-induced gingival overgrowth. J Clin Periodontol, 27(4): 217-223.

[20] Seymour RA (2006). Effects of medications on the periodontal tissues in health and disease. Perio 2000, 4: 120-129. 